\title{
ON ALGEBRAIC DIFFERENTIAL EQUATIONS OF GAMMA FUNCTION AND RIEMANN ZETA FUNCTION
}

\author{
Feng Lü \\ China University of Petroleum, College of Science \\ Qingdao, Shandong, 266580, P.R. China; lvfeng18@gmail.com
}

\begin{abstract}
Due to Voronin's universality theorem and Riemann-von Mangoldt formula, this paper concerns the problem of algebraic differential independence between the gamma function $\Gamma$ and the function $f(\zeta)$, where $\zeta$ is the Riemann zeta function and $f$ is a function with at least one zero-point. It is showed that $\Gamma$ and $f(\zeta)$ cannot satisfy any nontrivial distinguished differential equation with meromorphic coefficients $\phi$ having Nevanlinna characteristic satisfying $T(r, \phi)=o(r)$ as $r \rightarrow \infty$.
\end{abstract}

\section{Introduction and main result}

The paper is devoted to studying the question of whether the gamma function $\Gamma$ and Riemann zeta function $\zeta$, are algebraically independent or not. In 1886, Hölder [8] proved a profound theorem which states that the gamma function does not satisfy any non-trivial algebraic differential equation whose coefficients are rational functions in C. Later, Bank and Kaufman [1] generalized the above theorem to coefficients being meromophic functions $\phi$ with Nevanlinna characteristic satisfying $T(r, \phi)=o(r)$. The question of the differential independence of $\zeta$ was introduced by Hilbert. He [7] conjectured that $\zeta$ and other functions of the same type do not satisfy algebraic differential equations with rational functions. The problem was solved in $[17,18]$. It is known that $\zeta$ is associated with $\Gamma$ by the Riemann functional equation

$$
\zeta(1-s)=2^{1-s} \pi^{-s} \cos \left(\frac{1}{2} \pi s\right) \Gamma(s) \zeta(s) .
$$

It is natural to ask whether the functions $\Gamma$ and $\zeta$ are related by any nontrivial algebraic differential equation. In 2007, Markus [15] deduced that $\Gamma$ and the composition function $\zeta(\sin (2 \pi z))$ are differential independent over $\mathbf{C}$. He conjectured that $\Gamma$ is not a solution of any non-trivial algebraic differential equation-even allowing coefficients that are differential polynomials in $\zeta$. Recently, Li and Ye $[10,11]$ have done some efforts on this question. They proved that $\zeta$ is not a solution of any non-trivial algebraic differential equation-even allowing coefficients that are polynomials in $\Gamma, \Gamma^{\prime}$ and $\Gamma^{\prime \prime}$. The special case of algebraic independent question of $\Gamma$ and $\zeta$ is solved by $\mathrm{Li}$ and Ye in [11], Liao and Yang in [13], respectively. They proved that $\Gamma$ and $\zeta$ cannot satisfy nonzero polynomial equation $P(u, v, s)=0$. More generally, making use of the properties of $\Gamma$ and $\zeta, \mathrm{Li}$ and Ye [12] also showed that $P\left(s, \Gamma, \Gamma^{\prime}, \cdots, \Gamma^{(n)}, \zeta\right) \not \equiv 0$ in $\mathbf{C}$ for any nontrivial distinguished polynomial $P$ whose coefficients can be allowed to be any polynomials of $\zeta$ over $\mathbf{C}$, over the ring of polynomials or, more generally,

https://doi.org/10.5186/aasfm.2019.4455

2010 Mathematics Subject Classification: Primary 34M15, 11M06, 33B15, 30D30.

Key words: Voronin's university theorem, the Riemann zeta function, the gamma function, algebraic differential equation.

The research was supported by NNSF of China Project No. 11601521, and the Fundamental Research Fund for Central Universities in China Project No. 18CX02048A. 
over the class $L_{\delta}$ (see [12, Definition 1]), where

$$
P\left(s, u_{0}, u_{1}, \cdots, u_{n}, v\right)=\sum_{k=0}^{m} P_{k}\left(s, u_{0}, u_{1}, \cdots, u_{n}\right) v^{k} .
$$

Here, the distinguished polynomial $P$ is defined as follows.

Definition 1. Let $I=\left(i_{0}, i_{1}, \cdots, i_{n}\right)$ be a multi-index with $|I|=i_{0}+i_{1}+\cdots+i_{n}$. A polynomial in the variables $u_{0}, u_{1}, \cdots, u_{n}$ with functional coefficients $a_{I}$ can be always written as

$$
P\left(u_{0}, u_{1}, \cdots, u_{n}\right)=\sum_{I \in \Lambda} a_{I}(s) u_{0}^{i_{0}} u_{1}^{i_{1}} \cdots u_{n}^{i_{n}}
$$

where $\Lambda$ is an index set. We call $P$ a distinguished polynomial in $u_{0}, u_{1}, \cdots, u_{n}$ or simply a distinguished polynomial, if the index set $\Lambda$ satisfies that $\left|I_{i}\right| \neq\left|I_{j}\right|$ for distinct indices $I_{i}, I_{j}$ in $\Lambda$.

Motivated by the above results, it is natural to ask whether

$$
P\left(s, \Gamma, \Gamma^{\prime}, \cdots, \Gamma^{(n)}, \zeta\right) \not \equiv 0
$$

in $\mathbf{C}$ for any nontrivial distinguished polynomial $P$ whose meromorphic coefficients $\phi$ satisfy $T(r, \phi)=o(r)$. The problem has been solved by the present author in [14]. In this paper, we still pay attention to this kind of algebraically independent problem. In fact, due to the Voronin's universality theorem, Riemann-von Mangoldt formula and minimum modulus theorem, we derive the following result.

Theorem 1. Let $f(s)(s \in \mathbf{C})$ be a function with at least one zero-point and

$$
P\left(s, u_{0}, u_{1}, \cdots, u_{n}, v\right)=\sum_{k=0}^{m} P_{k}\left(s, u_{0}, u_{1}, \cdots, u_{n}\right) v^{k},
$$

where $P_{k}$, not all identically zero, are distinguished polynomials with meromorphic coefficients $\phi$ satisfying $T(r, \phi)=o(r)$. Then for $s \in \mathbf{C}$

$$
P\left(s, \Gamma, \Gamma^{\prime}, \cdots, \Gamma^{(n)}, f(\zeta)\right) \not \equiv 0 .
$$

Remark 1. It is pointed out that by the universality property of $\zeta$ (which is defined in Lemma 2) we handle the case that $f(s)$ has a zero $c(\neq 0)$. Observe that $\zeta^{(k)}$ and $\sum_{n=1}^{k} a_{n} \zeta^{(n)}$ with $a_{n} \neq 0$ have the strong universality property. Then, the same argument of Theorem 1 can yield the conclusion of Theorem 1 still holds if the function $\zeta$ is replaced by the function $g$ even $c=0$, where $g=\zeta^{(k)}$ or $\sum_{n=1}^{k} a_{n} \zeta^{(n)}$. It means that $P\left(s, \Gamma, \Gamma^{\prime}, \cdots, \Gamma^{(n)}, f(g)\right) \not \equiv 0$ in $\mathbf{C}$.

Remark 2. It is well-known that Dirichlet $L$-function $L(s, \chi)$ also has the universality property. So if $f(s)$ has a nonzero zero-point, then the same process of Theorem 1 yields $P\left(s, \Gamma, \Gamma^{\prime}, \cdots, \Gamma^{(n)}, f(L(s, \chi))\right) \not \equiv 0$. We point out that the distribution of zeros of $\zeta$ which lie in the line $\operatorname{Re}=\frac{1}{2}$ is essential to the proof of Theorem 1 when $f(s)=0$ only has a root which is zero. However, we don't know the distribution of zeros of $L(s, \chi)$ which lie in the line $\operatorname{Re}=\frac{1}{2}$. Therefore, we are not sure whether the conclusion holds or not if the equation $f(s)=0$ only has a root which is zero. Similar conclusions can be obtained for the Hecke $L$-functions, $L$-functions associated to newforms and many other $L$-functions.

Since a nontrivial polynomial $P(s, u, v)$ can be written into the form $P(s, u, v)=$ $\sum_{k=0}^{m} P_{k}(s, u) v^{k}$ with $P_{k}(s, u)$ being distinguished polynomial in one argument $u$, we have the following. 
Corollary. Let $f(s)(s \in \mathbf{C})$ be a function with at least one zero-point. Then in $\mathbf{C}, P\left(s, \Gamma^{(n)}, f(\zeta)\right) \not \equiv 0$ for any nontrivial polynomial $P(s, u, v)$ with meromorphic coefficients $\phi$ satisfying $T(r, \phi)=o(r)$ as $r \rightarrow \infty$.

To prove the main result, we will employ the following results and notations. For a meromorphic function $f$, we define $Z_{f}$ the set of all the zeros of $f$, counting multiplicities. Denote by $n\left(r, Z_{f}\right)$ the number of the points $Z_{f} \cap\{|s|<r\}$. The following lemma is called minimum modulus theorem, (see e.g. [2, p.362, 4.5.14]).

Lemma 1. Let $f(s)$ be holomorphic in the disc $B(0,2 e R)$ and continuous in the closure of the disc. Assume that $f(0)=1$ and let $\theta>0$ be such that $0<\theta<\frac{3 e}{2}$. Then, in the disc $|s| \leq R$, and outside a collection of closed disc $O_{1}, \cdots, O_{p}(p \leq q=$ $\left.n\left(R, Z_{f}\right)\right)$ the sum of whose radii does not exceed $4 \theta R$, we have

$$
\log |f(s)| \geq-\left(2+\log \frac{3 e}{2 \theta}\right) \log M(2 e R, f) .
$$

In the 1970's, Voronin [21] discovered the remarkable fact that Riemann zeta function $\zeta$ has a universality property, stated below, (which is Voronin's university theorem). It plays a essential role in the proof of Theorem 1.

Lemma 2. Let $0<r<\frac{1}{4}$ be a real number. Let $\varphi$ be a non-vanishing continuous function in $|s| \leq r$, that is analytic in the interior. Then, for any $\epsilon>0$,

$$
\liminf _{T \rightarrow \infty} \frac{1}{T} \operatorname{meas}\left\{T \leq \tau \leq 2 T: \max _{|s| \leq t}\left|\zeta\left(\frac{3}{4}+i \tau+s\right)-\varphi(s)\right|<\epsilon\right\}>0,
$$

where meas $\{A\}$ is Lebesgue measure of the measurable subset $A$ of $\mathbf{R}$.

Remark 3. There are several extensions of Voronin's universality theorem, for example to domain more general than compact discs (such as any compact set $K$ contained in the strip $1 / 2<\operatorname{Re}(s)<1$ and with connected complement), or to more general $L$-functions, such as Dirichlet $L$-functions, Hecke $L$-functions. Furthermore, it turns out that there exist also a lot of zeta-functions with strong universality property, where the attribute strong means that also functions having zeros can be approximated. For a complete history of this subject, we refer the reader to [16]. Since $\zeta$ does not have strong universality property, we below try to derive a result, which plays the same role as the Voronin's universality theorem in the proof of Theorem 1.

Lemma 3. For any $\eta>0$, the set $E_{\eta}(T)$ is defined as

$$
E_{\eta}(T)=\left\{\tau \in[T, 4 T] \cap[s-\eta, s+\eta]: \zeta\left(\frac{1}{2}+i s\right)=0, s>0\right\} .
$$

Then

$$
\liminf _{T \rightarrow \infty} \frac{\operatorname{meas} E_{\eta}(T)}{T}>0
$$

Proof of Lemma 3. Suppose, on the contrary, $\liminf _{T \rightarrow \infty} \frac{\text { meas } E_{\eta}(T)}{T}=0$. Then, there exist $\eta>0$ and a subsequence $\left\{T_{k}\right\}$ such that

$$
\text { meas } E_{\eta}\left(T_{k}\right)=o\left(T_{k}\right) \text { as } T_{k} \rightarrow \infty \text {. }
$$

Set $E_{\eta}\left(T_{k}\right)=\bigcup_{i=1}^{n_{k}}\left[a_{i}, a_{i}+\Delta_{i}\right]$ the disjoint intervals in $\left[T_{k}, 4 T_{k}\right]$. Note that for $T \geq T_{0}$,

$$
N(T)=\frac{T}{2 \pi} \log \frac{T}{2 \pi}-\frac{T}{2 \pi}+O(\log T),
$$


where $N(T)$ denotes the number of the zeros of $\zeta$ which lie in the domain $\{s: 0<$ $\operatorname{Re} s<1,0<\operatorname{Im} s<T\}$. Suppose that $T_{k} \geq T_{0}$. Then,

$$
\begin{aligned}
N\left(a_{i}+\Delta_{i}\right)-N\left(a_{i}\right)= & \frac{a_{i}+\Delta_{i}}{2 \pi} \log \frac{a_{i}+\Delta_{i}}{2 \pi}-\frac{a_{i}+\Delta_{i}}{2 \pi}+O\left(\log a_{i}+\Delta_{i}\right) \\
& -\left[\frac{a_{i}}{2 \pi} \log \frac{a_{i}}{2 \pi}-\frac{a_{i}}{2 \pi}+O\left(\log a_{i}\right)\right] \\
\leq & \frac{\Delta_{i}}{2 \pi} \log \frac{a_{i}+\Delta_{i}}{2 \pi}+O\left(\log \left(a_{i}+\Delta_{i}\right)\right) \\
\leq & \frac{\Delta_{i}}{2 \pi} \log \frac{a_{i}+\Delta_{i}}{2 \pi}+M \log \left(a_{i}+\Delta_{i}\right) \leq\left(\frac{\Delta_{i}}{2 \pi}+M\right) \log 4 T_{k},
\end{aligned}
$$

where $M$ is a fixed positive constant. Define the set $N_{0}(T)$ as

$$
N_{0}(T)=\sharp\left\{\rho=1 / 2+i \gamma: 0<\gamma<T, \zeta(\rho)=0, \zeta^{\prime}(\rho) \neq 0\right\},
$$

where $\sharp E$ denotes the number of the elements in the set $E$. Note that $n_{k} \leq$ $\frac{\text { meas } E_{\eta}\left(T_{k}\right)}{2 \eta}+2$. Thus,

$$
\begin{aligned}
N_{0}\left(4 T_{k}\right) & \leq \sum_{i=1}^{n_{k}}\left[N\left(a_{i}+\Delta_{i}\right)-N\left(a_{i}\right)\right]+N\left(T_{k}\right) \leq \sum_{i=1}^{n_{k}}\left[\left(\frac{\Delta_{i}}{2 \pi}+M\right) \log 4 T_{k}\right]+N\left(T_{k}\right) \\
& \leq\left(\frac{\text { meas } \left.E_{\eta}\left(T_{k}\right)\right)}{2 \pi}+n_{k} M\right) \log 4 T_{k}+N\left(T_{k}\right) \\
& \leq\left[\frac{\left.\operatorname{meas} E_{\eta}\left(T_{k}\right)\right)}{2 \pi}+\left(\frac{\operatorname{meas} E_{\eta}\left(T_{k}\right)}{2 \eta}+2\right) M\right] \log 4 T_{k}+N\left(T_{k}\right) \\
& =o\left(T_{k} \log 4 T_{k}\right)+N\left(T_{k}\right)=\frac{T_{k}}{2 \pi} \log \frac{T_{k}}{2 \pi}+o\left(T_{k} \log T_{k}\right) .
\end{aligned}
$$

On the other hand, we know that $N_{0}(T) \geq \frac{2}{5} N(T)$ for $T$ large enough, which is given by Conrey in [4, Theorem 1]. Thus,

$$
N_{0}\left(4 T_{k}\right) \geq \frac{2}{5} N\left(4 T_{k}\right)=\frac{2}{5} \times 4 N\left(T_{k}\right)+o\left(T_{k} \log T_{k}\right) \geq \frac{8}{5} \frac{T_{k}}{2 \pi} \log \frac{T_{k}}{2 \pi}+o\left(T_{k} \log T_{k}\right),
$$

a contradiction. Thus, the lemma is proved.

Proof of theorem 1. Due to the ideas in $[10,11,12,14]$, we will prove Theorem 1. The polynomial $P\left(s, u_{0}, \cdots, u_{n}, v\right)$ may be written as the following form

$$
\begin{aligned}
P\left(s, u_{0}, \cdots, u_{n}, v\right)= & v^{m} \sum_{I \in \Lambda_{m}} a_{m, I} u_{0}^{i_{0}} u_{1}^{i_{1}} \cdots u_{n}^{i_{n}}+v^{m-1} \sum_{I \in \Lambda_{m}-1} a_{m-1, I} u_{0}^{i_{0}} u_{1}^{i_{1}} \cdots u_{n}^{i_{n}}+ \\
& \cdots+v \sum_{I \in \Lambda_{1}} a_{1, I} u_{0}^{i_{0}} u_{1}^{i_{1}} \cdots u_{n}^{i_{n}}+\sum_{I \in \Lambda_{0}} a_{0, I} u_{0}^{i_{0}} u_{1}^{i_{1}} \cdots u_{n}^{i_{n}}
\end{aligned}
$$

where $m$ is the highest power of $v$ in the polynomial $P$ and $\Lambda_{j}$ 's are index sets. Obviously, all the coefficients $a_{i, I}$ are either identically zero in $\mathbf{C}$ or nonzero meromorphic functions with $T\left(r, a_{i, I}\right)=o(r)$. On the contrary, suppose that $\Gamma, \Gamma^{\prime}, \cdots, \Gamma^{(n)}, f(\zeta)$ 
satisfy $P\left(s, u_{0}, \cdots, u_{n}, f(\zeta)\right)=0$ in $\mathbf{C}$. That is

$$
\begin{aligned}
P\left(s, \Gamma, \Gamma^{\prime}, \cdots, \Gamma^{(n)}, f(\zeta)\right)= & f(\zeta)^{m} \sum_{I \in \Lambda_{m}} a_{m, I} \Gamma^{i_{0}}\left(\Gamma^{\prime}\right)^{i_{1}} \cdots\left(\Gamma^{(n)}\right)^{i_{n}} \\
& +f(\zeta)^{m-1} \sum_{I \in \Lambda_{m-1}} a_{m-1, I} \Gamma^{i_{0}}\left(\Gamma^{\prime}\right)^{i_{1}} \cdots\left(\Gamma^{(n)}\right)^{i_{n}}+ \\
& \cdots+f(\zeta) \sum_{I \in \Lambda_{1}} a_{1, I} \Gamma^{i_{0}}\left(\Gamma^{\prime}\right)^{i_{1}} \cdots\left(\Gamma^{(n)}\right)^{i_{n}} \\
& +\sum_{I \in \Lambda_{0}} a_{0, I} \Gamma^{i_{0}}\left(\Gamma^{\prime}\right)^{i_{1}} \cdots\left(\Gamma^{(n)}\right)^{i_{n}}=0 .
\end{aligned}
$$

We will prove that all the coefficients $a_{i, I}$ in (1.1) are identically zero in $\mathbf{C}$ for all possible $i, I$. This, of course, contradicts to the assumption of the theorem.

Firstly, we will show $a_{0, I} \equiv 0$ in the last sum of (1.1). Suppose that the index set $\Lambda_{0}$ contains $t$ indices $I_{1}, I_{2}, \cdots, I_{t}$. Based on the increasing order, we list all these indices as $\left|I_{1}\right|<\left|I_{2}\right|<\cdots<\left|I_{t}\right|$. So, the last sum of (1.1) can be written as

$$
\sum_{j=1}^{t} a_{0, I_{j}} \Gamma^{i_{0}}\left(\Gamma^{\prime}\right)^{i_{1}} \cdots\left(\Gamma^{(n)}\right)^{i_{n}}
$$

Suppose that $a_{0, I_{1}} \not \equiv 0$. We will derive a contradiction below. By the assumption, we know that $f(s)$ has a zero, say $c$. Suppose that there exists a sequence $\left\{s_{l}=x_{l}+i y_{l}\right\}$ such that $x_{l} \in[0,1], y_{l} \rightarrow+\infty$ and $\zeta\left(s_{l}\right)=c$. Meanwhile, assume $s_{l}$ is not the pole and zero of the coefficients of the differential polynomial $P$. By taking a subsequence if necessary, we may assume that $x_{l} \rightarrow u_{0} \in[0,1]$ as $l \rightarrow \infty$.

Note that $f\left(\zeta\left(s_{l}\right)\right)=0$ and $s_{l}$ is not the pole and zero of the coefficients of the differential polynomial $P$. We obtain, from (1.1) and (1.2), that

$$
\sum_{j=1}^{t} a_{0, I_{j}} \Gamma^{i_{0}}\left(\Gamma^{\prime}\right)^{i_{1}} \cdots\left(\Gamma^{(n)}\right)^{i_{n}}\left(s_{l}\right)=0 .
$$

In view of $a_{0, I_{1}} \not \equiv 0$, one can rewrite the above equation as

$$
\sum_{j=2}^{t} \frac{a_{0, I_{j}}}{a_{0, I_{1}}}\left(\frac{\Gamma^{\prime}}{\Gamma}\right)^{i_{1}} \cdots\left(\frac{\Gamma^{(n)}}{\Gamma}\right)^{i_{n}} \Gamma^{\left|I_{j}\right|-\left|I_{1}\right|}\left(s_{l}\right)=-\left(\frac{\Gamma^{\prime}}{\Gamma}\right)^{i_{1}} \ldots\left(\frac{\Gamma^{(n)}}{\Gamma}\right)^{i_{n}}\left(s_{l}\right) .
$$

Suppose that $\phi$ is a coefficient of the differential polynomial $P$. Then, $T(r, \phi)=o(r)$. Clearly, $N(r, \phi) \leq T(r, \phi)=o(r)$, where $N(r, \phi)$ is the Nevanlinna counting function of the zeros of $\phi$ and defined as

$$
N(r, \phi)=\int_{0}^{r} \frac{n\left(t, Z_{\phi}\right)-n\left(0, Z_{\phi}\right)}{t} d t+n\left(0, Z_{\phi}\right) \log r .
$$

Therefore, for any $\varepsilon>0$, there exists a positive constant $r_{1}$ such that $N(r, \phi) \leq \varepsilon r$ for $r>r_{1}$. Furthermore,

$$
\varepsilon(2 r) \geq N(2 r, \phi) \geq \int_{r}^{2 r} \frac{n\left(t, Z_{\phi}\right)}{t} d t \geq \frac{n\left(r, Z_{\phi}\right)}{2 r} r=\frac{n\left(r, Z_{\phi}\right)}{2},
$$

which implies that $n\left(r, Z_{\phi}\right)=o(r)$. Similarly, one has that $n\left(r, Z_{\frac{1}{\phi}}\right)=o(r)$. Here, $n\left(r, Z_{\frac{1}{\phi}}\right)$ denotes the number of the poles of $\phi$ in the disc $|s|<r$, counting multiplicities. 
Now, assume that $G$ is the set of the poles and zeros of all the coefficients $a_{j, I}$ of the polynomial $P$. Then, it follows from the above discussions that

$$
n(r, G)=o(r)
$$

Set

$$
g(s)=\prod_{\rho \in G}\left(1-\frac{s^{2}}{\rho^{2}}\right) \quad \text { and } \quad f_{j}(s)=\frac{a_{0, I_{j}}}{a_{0, I_{1}}}, \quad j=2, \cdots, t
$$

It is easy to check that the infinite product $g(s)$ converges to an entire function (this will be showed in (1.8) below). Then, multiplying $e^{\frac{\pi}{4} y_{l}} g\left(s_{l}\right)$ on both sides of (1.4) yields

$$
\begin{aligned}
& e^{\frac{\pi}{4} y_{l}} \sum_{j=2}^{t} g\left(s_{l}\right) f_{j}\left(s_{l}\right)\left(\frac{\Gamma^{\prime}}{\Gamma}\right)^{i_{1}} \cdots\left(\frac{\Gamma^{(n)}}{\Gamma}\right)^{i_{n}} \Gamma^{\left|I_{j}\right|-\left|I_{1}\right|}\left(s_{l}\right) \\
& =-e^{\frac{\pi}{4} y_{l}} g\left(s_{l}\right)\left(\frac{\Gamma^{\prime}}{\Gamma}\right)^{i_{1}} \cdots\left(\frac{\Gamma^{(n)}}{\Gamma}\right)^{i_{n}}\left(s_{l}\right) .
\end{aligned}
$$

In the following, we will estimate the growths of some terms in (1.6) such as

$$
\frac{\Gamma^{(q)}(s)}{\Gamma(s)}, g(s), \text { and } g(s) f_{j}(s)
$$

where $q$ is a positive integer. We consider the next steps.

Step 1. Estimate the modulus of $\frac{\Gamma^{(q)}(s)}{\Gamma(s)}$. We employ the following asymptotic formula (see e.g. 151 in [20]), which is called Stirling formula,

$$
|\Gamma(s)|=|\Gamma(x+i y)|=\sqrt{2 \pi}|x|^{y-1 / 2} e^{-\frac{\pi}{2}|y|}\left[1+O\left(\frac{1}{y}\right)\right], \quad\left(|y| \rightarrow \infty, \sigma_{1} \leq \operatorname{Re} s \leq \sigma_{2}\right),
$$

where $\sigma_{1}$ and $\sigma_{2}$ are two real constants. In [12], we know that for any positive integer $q$,

$$
\Gamma^{(q)}(s)=(1+o(1))(\log s)^{q} \Gamma(s)
$$

uniformly for all $s \in \mathbf{C} \backslash\{s:|\arg s-\pi| \leq \epsilon\}$ with any small $\epsilon>0$.

Step 2. Estimate the growths of $g(s)$ and $g(s) f_{j}(s)$. With the same way in [14], we can deduce that

$$
|g(s)| \leq e^{A \varepsilon|s|}, \quad\left|f_{j}(s) g(s)\right| \leq e^{B \varepsilon|s|}
$$

where $A$ and $B$ are two positive constants. For the completeness of the process, we give the proof below.

Observing the fact (1.5), for arbitrary small $\varepsilon>0$, we below assume that $n(r, G) \leq \varepsilon r$ for $r \geq r_{0}$, where $r_{0}$ is a positive constant. By making use of the 
method in [9], one has,

$$
\begin{aligned}
\log |g(s)| & =\log \left|\prod_{\rho \in G}\left(1-\frac{s^{2}}{\rho^{2}}\right)\right| \leq \sum_{\rho \in G} \log \left(1+\left|\frac{s^{2}}{\rho^{2}}\right|\right) \\
& =\int_{0}^{\infty} \log \left(1+\left|\frac{s^{2}}{r^{2}}\right|\right) d(n(r, G)) \\
& =\left.\log \left(1+\left|\frac{s^{2}}{r^{2}}\right|\right) n(r, G)\right|_{0} ^{\infty}+2|s|^{2} \int_{0}^{\infty} \frac{n(r, G)}{r\left(r^{2}+|s|^{2}\right)} d r \\
& =2|s|^{2}\left[\int_{0}^{r_{0}} \frac{n(r, G)}{r\left(r^{2}+|s|^{2}\right)} d r+\int_{r_{0}}^{\infty} \frac{n(r, G)}{r\left(r^{2}+|s|^{2}\right)} d r\right] \\
& \leq 2|s|^{2}\left\{\frac{1}{|s|^{2}} \int_{0}^{r_{0}} \frac{n(r, G)}{r} d r+\int_{r_{0}}^{\infty} \frac{\varepsilon r}{r\left(r^{2}+|s|^{2}\right)} d r\right\} \leq A \varepsilon|s|,
\end{aligned}
$$

where $A$ is a positive constant. Thus,

$$
\log M(r, g) \leq A \varepsilon|s|,
$$

where $M(r, g)=\max _{|s|=r}\{|g(s)|\}$, the maximum modulus of $g$ on $|s|=r$. Set $E=\left\{\varsigma:\left|g\left(r e^{i \varsigma}\right)\right|>1\right\}$. Then,

$$
\begin{aligned}
T(r, g) & =m(r, g)=\frac{1}{2 \pi} \int_{0}^{2 \pi} \log ^{+}\left|g\left(r e^{i \varsigma}\right)\right| d \varsigma \\
& =\frac{1}{2 \pi} \int_{\varsigma \in E} \log ^{+}\left|g\left(r e^{i \varsigma}\right)\right| d \varsigma=\frac{1}{2 \pi} \int_{\varsigma \in E} \log \left|g\left(r e^{i \varsigma}\right)\right| d \varsigma \\
& \leq \frac{1}{2 \pi} \int_{\varsigma \in E} A \varepsilon\left|r e^{i \varsigma}\right| d \varsigma \leq A \varepsilon r .
\end{aligned}
$$

Applying the first main theorem in Nevanlinna theory to the function $a_{0, I_{j}}$, one has

$$
\begin{aligned}
T\left(r, f_{j}(s)\right) & =T\left(r, \frac{a_{0, I_{j}}}{a_{0, I_{1}}}\right) \leq T\left(r, a_{0, I_{j}}\right)+T\left(r, \frac{1}{a_{0, I_{1}}}\right)+O(1) \\
& \leq T\left(r, a_{0, I_{j}}\right)+T\left(r, a_{0, I_{1}}\right)+O(1)=o(r) .
\end{aligned}
$$

Recall the well-known result (see, e.g., [6]) in Nevanlinna theory

$$
\log ^{+} \max _{|s|=r}\{|f(s)|\} \leq \frac{R+r}{R-r} T(R, f)
$$

for $R>r>0$, if $f$ is entire. Obviously, $f_{j}(s) g(s)$ is an entire function. Then, we have for $|s|=r$ and $R=2 r$

$$
\begin{aligned}
\log \left|f_{j}(s) g(s)\right| & \leq \log \max _{|s|=r}\left\{\left|f_{j} g\right|\right\} \leq \log ^{+} \max _{|s|=r}\left\{\left|f_{j} g\right|\right\} \leq \frac{R+r}{R-r} T\left(R, f_{j} g\right) \\
& \leq 3 T\left(2 r, f_{j} g\right) \leq 3\left[T\left(2 r, f_{j}\right)+T(2 r, g)+O(1)\right] \leq B \varepsilon|s|,
\end{aligned}
$$

where $B$ is a positive constant. It shows (for $j=2, \cdots, t$ ) that

$$
\left|f_{j}(s) g(s)\right| \leq e^{B \varepsilon|s|} .
$$

Step 3. We shall prove the following proposition.

Proposition. For $\varepsilon>0$ small enough, there exist a sequence $\left\{s_{l}\right\}$ in the domain $D:=\left\{s: \frac{1}{2} \leq \operatorname{Re} s \leq 1, \operatorname{IM} s>0\right\}$ such that

$$
\zeta\left(s_{l}\right)=c \quad \text { and } \quad g\left(s_{l}\right)>e^{-C \varepsilon\left|s_{l}\right|},
$$


where $C$ is a fixed positive constant. Meanwhile, $s_{l}$ is not the pole and zero of the coefficients of the differential polynomial $P$.

Proof of Proposition. We will employ the lemmas 1-3 to deal with this proposition. Set $h \geq 0$ be an integer. Note that $g(0)=1$. Then, for $2^{h} \leq|s|=r \leq R=2^{h+2}$ and $h$ large enough, applying Lemma 1 to the function $g$, one has, that

$$
\begin{aligned}
\log |g(s)| & \geq-\left(2+\log \frac{3 e}{2 \theta}\right) \log M(2 e R, g) \geq-\left(2+\log \frac{3 e}{2 \theta}\right) A \varepsilon 2 e 2^{h+2} \\
& =-\left(2+\log \frac{3 e}{2 \theta}\right) A \varepsilon 2 e \cdot 2^{h} 4 \geq-\left(2+\log \frac{3 e}{2 \theta}\right) A \varepsilon 8 e \cdot r
\end{aligned}
$$

outside a collections of closed $p$ disks $\bigcup_{l=1}^{p} O_{l}\left(p \leq q=n\left(R, Z_{g}\right)\right)$ whose radii add up to at most $4 \theta R=4 \theta 2^{h+2}$. Next, we split into two cases.

Case 1. $c \neq 0$. Some ideas are based on [5]. Suppose that $K:=\left\{s:\left|s-\frac{3}{4}\right|<t\right\}$ and $t(<|c|)$ is a fixed positive number. Define the function $\varphi(s)=c+\left(s-\frac{3}{4}\right)$ in $K$. Obviously, $\varphi(s)$ is a non-vanishing analytic function in $K$. For $h$ (which is large enough) and $\frac{t}{2}$, it follows from Lemma 2 (Voronin's universality theorem) that meas $E_{0}>\omega 2^{h}$ where

$$
E_{0}=\left\{2^{h} \leq \tau \leq 2^{h+2}=R: \max _{s \in K}\left|\zeta\left(\frac{3}{4}+i \tau+s\right)-\varphi(s)\right|<\frac{t}{2}\right\},
$$

and $\omega$ is a fixed positive constant. We define the set $E_{1}$ as

$$
E_{1}=\left\{\tau: 0<\tau<R=2^{h+2} \text { and } d\left(\frac{3}{4}+i \tau, \bigcup_{l=1}^{p} O_{l}\right)<2 t\right\}
$$

where $d($,$) is the distant between a point and a set. Then, an elementary calculation$ yields

$$
\text { meas } E_{1} \leq 4 \theta R+4 t p \leq 4 \theta R+4 t n\left(R, Z_{g}\right) \leq 16(\theta+2 t \varepsilon) 2^{h} \text {. }
$$

Note that $\theta$ and $\varepsilon$ can be small enough. So take $\theta$ and $\varepsilon$ such that $16(\theta+2 t \varepsilon)<\omega$. Thus, there exists at lease one point $\tau_{1} \in E_{0} \backslash E_{1}$. Furthermore,

$$
\max _{s \in K}\left|\zeta\left(\frac{3}{4}+i \tau_{1}+s\right)-\varphi(s)\right|<\frac{t}{2} .
$$

Thus,

$$
\max _{s \in \partial K}\left|\zeta\left(\frac{3}{4}+i \tau_{1}+s\right)-c-(\varphi(s)-c)\right|<\frac{t}{2}<t=\max _{s \in \partial K}|\varphi(s)-c|,
$$

and an application of Rouché's theorem gives the existence of a $c$-point of $\zeta(s)$ inside $K+i \tau_{1}=\left\{s+i \tau_{1}: s \in K\right\}$, say $s_{h}$. Obviously, $s_{h} \notin \cup_{l=1}^{p} O_{l}$, since $\tau_{1} \notin E_{1}$. Therefore, by (1.12), one gives

$$
\log \left|g\left(s_{h}\right)\right|>-C \varepsilon\left|s_{h}\right|, \quad \text { with } C=-\left(2+\log \frac{3 e}{2 \theta}\right) A 8 e .
$$

Letting the integer $h$ increase to $\infty$, one can get a sequence $\left\{s_{h}\right\}$. Rewrite $s_{h}$ by $s_{l}$. Thus, we derive the desired conclusion of the proposition.

Case 2. $c=0$. Let $\eta=t$ in Lemma 3. Set

$$
E_{2}=\left\{\tau: 0<\tau<R=2^{h+2} \text { and } d\left(\frac{1}{2}+i \tau, \bigcup_{l=1}^{p} O_{l}\right)<2 t\right\} .
$$

Then, the same argument of Case 1 gives that there exists at least one point $\tau_{h} \in$ $E_{t}\left(2^{h}\right) \backslash E_{2}$. Thus, by the definition of $E_{t}\left(2^{h}\right)$, there exist a zero $s_{h}=\frac{1}{2}+i y_{h}$ of $\zeta$ such that $y_{h} \in\left[\tau_{h}-\eta, \tau_{h}+\eta\right]=\left[\tau_{h}-t, \tau_{h}+t\right]$. Obviously, $s_{h} \notin \bigcup_{l=1}^{p} O_{l}$. Similarly as Case 1 , we get the conclusion of this proposition. 
Step 4. We will finish the proof of Theorem 1. The above discussion yields that $s_{l}$ is not the zero and pole of the coefficients of differential polynomial $P$. Still substitute $s_{l}$ in (1.4). Choosing the point $\varepsilon$ such that $\frac{\pi}{4}-B \varepsilon>0$ and $\frac{\pi}{4}-C \varepsilon>0$. Then, the left side of (1.4) can be estimated as

$$
\begin{aligned}
e^{\frac{\pi}{4}} y_{l} & \left|g\left(s_{l}\right) f_{j}\left(s_{l}\right)\right|\left|\left(\frac{\Gamma^{\prime}}{\Gamma}\right)^{i_{1}} \cdots\left(\frac{\Gamma^{(n)}}{\Gamma}\right)^{i_{n}} \Gamma^{\left|I_{j}\right|-\left|I_{1}\right|}\left(s_{l}\right)\right| \\
\leq & e^{\frac{\pi}{4} y_{l}} e^{B \varepsilon\left|s_{l}\right|}\left|(1+o(1))(\log s)^{i_{1}+2 i_{2}+\cdots+n i_{n}} \Gamma^{\left|I_{j}\right|-\left|I_{1}\right|}\left(s_{l}\right)\right| \\
\leq & (1+o(1))(\sqrt{2 \pi})^{\left|I_{j}\right|-\left|I_{1}\right|} e^{\frac{\pi}{4} y_{l}} e^{B \varepsilon\left|y_{l}\right|}\left|\left(\log s_{l}\right)^{i_{1}+2 i_{2}+\cdots+n i_{n}}\right| \\
& \cdot\left|x_{l}\right|^{\left(y_{l}-1 / 2\right)\left(\left|I_{j}\right|-\left|I_{1}\right|\right)} e^{-\frac{\pi}{2} y_{l}}\left[1+O\left(\frac{1}{y_{l}}\right)\right] \\
\leq & (1+o(1))(\sqrt{2 \pi})^{\left|I_{j}\right|-\left|I_{1}\right|} e^{-\left(\frac{\pi}{4}-B \varepsilon\right) y_{l}} \\
& \cdot\left|\left(\log s_{l}\right)^{i_{1}+2 i_{2}+\cdots+n i_{n}}\right| u_{0}^{\left(y_{l}-1 / 2\right)\left(\left|I_{j}\right|-\left|I_{1}\right|\right)}\left[1+O\left(\frac{1}{y_{l}}\right)\right] \rightarrow 0, \text { as } l \rightarrow \infty .
\end{aligned}
$$

So, the right side of (1.4) satisfies

$$
\left|-e^{\frac{\pi}{4} y_{l}} g\left(s_{l}\right)\left(\frac{\Gamma^{\prime}}{\Gamma}\right)^{i_{1}} \ldots\left(\frac{\Gamma^{(n)}}{\Gamma}\right)^{i_{n}}\left(s_{l}\right)\right| \rightarrow 0, \text { as } l \rightarrow \infty .
$$

On the other hand, by $\frac{\pi}{4}-C \varepsilon>0$, one has

$$
\begin{aligned}
& \left|-e^{\frac{\pi}{4} y_{l}} g\left(s_{l}\right)\left(\frac{\Gamma^{\prime}}{\Gamma}\right)^{i_{1}} \cdots\left(\frac{\Gamma^{(n)}}{\Gamma}\right)^{i_{n}}\left(s_{l}\right)\right| \\
& \geq e^{\frac{\pi}{4} y_{l}} e^{-C \varepsilon\left|s_{l}\right|}(1+o(1))\left|\left(\log s_{l}\right)^{i_{1}+2 i_{2}+\cdots+n i_{n}}\right| \\
& =e^{\left(\frac{\pi}{4}-C \varepsilon\right)\left|y_{l}\right|}(1+o(1))\left|\left(\log s_{l}\right)^{i_{1}+2 i_{2}+\cdots+n i_{n}}\right| \rightarrow \infty, \text { as } l \rightarrow \infty .
\end{aligned}
$$

This contradicts (1.15). Therefore,

$$
a_{0, I_{1}} \equiv 0
$$

Note that $a_{0, I_{1}}$ is identically zero, the expression (1.2) reduces to

$$
\sum_{j=2}^{t} a_{0, I_{j}} \Gamma^{i_{0}}\left(\Gamma^{\prime}\right)^{i_{1}} \cdots\left(\Gamma^{(n)}\right)^{i_{n}}\left(s_{l}\right)=0 .
$$

This is the same form as (1.2), except that $j$ starts from 2 now. The exact same argument as above shows that $a_{0, I_{2}}$ is identically zero. Repeating this argument, we get all the coefficients $a_{0, I_{j}}$ are identically zero. Therefore, (1.1) becomes

$$
\begin{aligned}
P\left(s, \Gamma, \Gamma^{\prime}, \cdots, \Gamma^{(n)}, f(\zeta)\right)= & f(\zeta)^{m-1} \sum_{I \in \Lambda_{m}} a_{m, I} \Gamma^{i_{0}}\left(\Gamma^{\prime}\right)^{i_{1}} \cdots\left(\Gamma^{(n)}\right)^{i_{n}} \\
& +f(\zeta)^{m-2} \sum_{I \in \Lambda_{m-1}} a_{m-1, I} \Gamma^{i_{0}}\left(\Gamma^{\prime}\right)^{i_{1}} \cdots\left(\Gamma^{(n)}\right)^{i_{n}}+ \\
& \cdots+\sum_{I \in \Lambda_{1}} a_{1, I} \Gamma^{i_{0}}\left(\Gamma^{\prime}\right)^{i_{1}} \cdots\left(\Gamma^{(n)}\right)^{i_{n}}
\end{aligned}
$$

which is the same form as (1.1), except that the highest power of $f(\zeta)$ is $m-1$ now. Thus, repeating the above process, one has all the coefficients of the polynomial identically zero, which contradicts the assumption. 
This completes the proof of Theorem 1.

Acknowledgment. The author would like to thank the referee for helpful suggestions.

\section{References}

[1] Bank, S., and R. Kaufman: An extension of Hölder's theorem concerning the gamma function. - Funkcial. Ekvac. 19, 1976, 53-63.

[2] Berenstein, C., and R. Gay: Complex variables. - Grad. Texts in Math., Springer-Verlag, 1991.

[3] Chinang, Y., and S. Feng: Difference independence of the Riemann zeta function. - Acta Arith. 125, 2006, 317-329.

[4] Conrey, J.: More than two fifths of the zeros of the Riemann zeta-function are on the critical line. - J. Reine Angew. Math. 399, 1989, 1-26.

[5] Garunkštis, R., J. Grahl, and J. Steuding: Uniqueness theorems for $L$-functions. - Comment. Math. Univ. St. Pauli 60:1-2, 2011, 15-35.

[6] Hayman, W.: Meromorphic function. - Clarendon Press, Oxford, 1964.

[7] Hilbert, D.: Mathematische Probleme. - Arch. Math. Phys. 1, 1901, 44-63, 213-317.

[8] HöLDER, O.: Uber die Eigenschaft der $\Gamma$-Function, keiner algebraischen Differentialgleichung zu genügen. - Math. Ann. 28, 1887, 1-13.

[9] KI, H., and B. LI: On uniqueness in the extended selberg class of dirichlet series. - Proc. Amer. Math. Soc. 141, 2013, 4169-4173.

[10] LI, B., and Z. YE: Algebraic differential equations concerning the Riemann zeta function and the Euler gamma function. - Indiana Univ. Math. J. 59, 2010, 1405-1415.

[11] Li, B., and Z. YE: On algebraic differential properties of the Riemann $\zeta$-function and Euler T-function. - Complex Var. Elliptic Equ. 56, 2011, 137-145.

[12] Li, B., and Z. YE: Algebraic differential equations with functional coefficients concerning $\zeta$ and $\Gamma$. - J. Differential Equations 260, 2016, 1456-1464.

[13] LiaO, L., and C. YANG: On some new properties of the gamma function and the Riemann zeta-function. - Math. Nachr. 257, 2003, 59-66.

[14] LÜ, F.: A study on algebraic differential equations of Gamma function and Dirichlet series. J. Math. Anal. Appl. 462, 2018, 1195-1204.

[15] Markus, L.: Differential independence of $\Gamma$ and $\zeta$. - J. Dynam. Differential Equations, 19, $2007,133-154$.

[16] Matsumoto, K.: A survey on the theory of universality for zeta and $L$-functions. - In: Number Theory, Ser. Number Theory Appl. 11, World Sci. Publ., Hackensack, NJ, 2015, 95-144.

[17] Mordykhai-Boltovskoi, D.: On hypertranscendence of the function $\xi(x, s)$. - Izv. Politekh. Inst. Warsaw 2, 1914, 1-16.

[18] Ostrowski, A.: Über Dirichletsche Reihen und algebraische Differentialgleichungen. - Math. Z. 8, 1920, 241-298.

[19] Steuding, J.: Value distribution of $\mathcal{L}$-functions. - Lecture Notes in Math. 1877, SpringerVerlag, Berlin, 2007.

[20] Titchmarsh, E.: The theory of functions. Second edition. - Oxford Univ. Press, reprinted, 1968.

[21] Voronin, S.: A theorem on the distribution of values of the Riemann zeta-function. - Dokl. Akad. Nauk SSSR 221:4, 1975, 771. 